\title{
Eficacia de dos inhibidores de la fosfodiesterasa 5 combinados con ginkgo biloba en la disfunción eréctil en hombres con enfermedades crónicas
}

\author{
Efficacy of two phosphodiesterase 5 inhibitors combined with ginkgo \\ biloba in erectile dysfunction in men with chronic diseases
}

\author{
Espitia FJ', Orozco L ${ }^{2}$.
}

\author{
${ }^{1}$ Ginecología y Obstetricia, Universidad Militar Nueva Granada. \\ Uroginecología/FUCS - Hospital de San José, Unicamp, Brasil. \\ Máster en Sexología: Educación y asesoramiento sexual. Universidad \\ de Alcalá de Henares. \\ Servicio de Ginecología y Medicina Materno Fetal, Clínica La \\ Sagrada Familia, Armenia, Quindío, Colombia. \\ ${ }^{2}$ Medicina Interna, Universidad El Bosque, Fundación Santa Fe, \\ Bogotá, Colombia.
}

Fecha de recepción: 08/03/2109

Fecha de aceptación: 10/04/2109

\section{Resumen}

Objetivo: comparar la eficacia terapéutica de dos fármacos inhibidores selectivos de la 5-fosfodiesterasa en combinación con ginkgo biloba para el tratamiento de la disfunción eréctil, en hombres con enfermedades crónicas concomitantes.

Material y métodos: ensayo clínico controlado, enmascarado y aleatorizado; realizado entre 2013 y diciembre de 2016. Se incluyeron hombres mayores de 45 años con disfunción eréctil no psicógena en tratamiento por enfermedades crónicas. Las variables de interés primario fueron: puntaje del índice internacional de la función eréctil, frecuencia coital mensual, tiempo de respuesta del fármaco, porcentaje de satisfacción de la mejoría de la disfunción y efectos adversos. Las variables cuantitativas se presentan como variables continuas mediante medidas de tendencia central y dispersión.

Resultados: 22 hombres fueron tratados con tadalafilo más ginkgo biloba (grupo A) y 23 con vardenafilo más ginkgo biloba (grupo B); edad promedio $57,6 \pm 8,4$ años y 58,2 $\pm 7,8$ para cada grupo, respectivamente. El puntaje del índice internacional de la función eréctil inicial fue de 9,63 \pm 1,26 (grupo A) y 10,35 $\pm 1,59$ (grupo B); al final del estudio fue de 21,78 \pm 0,9 (grupo A) y $18,57 \pm 0,3$ (grupo B), $p<0,015$. Finalmente, el grupo A incrementó la actividad sexual de 2 encuentros sexuales al mes a 9, mientras el grupo B pasó de 2 a 6, $p<0,021$.

Conclusiones: la combinación tadalafilo más ginkgo biloba tiene una satisfactoria eficacia terapéutica sobre la mejora en la puntuación del índice internacional de la función eréctil, que favorece la frecuencia coital mensual, con tiempo de inicio de acción y menores efectos adversos.

Palabras clave: eficacia; disfunción eréctil; enfermedad crónica; hombres; terapéutica.

\section{Abstract}

Objective: To compare the therapeutic efficacy of two selective inhibitors of 5-phosphodiesterase in combination with ginkgo biloba for the treatment of erectile dysfunction in men with chronic concomitant diseases.

Material and methods: Controlled, masked and randomized clinical trial; made between july 2013 and december 2016. Men older than 45 years with non-psychogenic erectile dysfunction in treatment for chronic diseases were included. The variables of primary interest were: international index of erectile function score, monthly coital frequency, time of drug response, percentage of satisfaction of the improvement of the dysfunction and adverse effects. Quantitative variables are presented as continuous variables through measures of central tendency and dispersion.

Results: 22 men were treated with tadalafil plus ginkgo biloba (group A) and 23 with vardenafil plus ginkgo biloba (group $B$ ); mean age $57.6 \pm 8.4$ years and $58.2 \pm 7.8$ for each group, respectively. The score of the initial international index of erectile function was $9.63 \pm 1.26$ (group A) and $10.35 \pm 1.59$ (group B); at the end of the study it was $21.78 \pm 0.9$ (group $A$ ), and 18.57 \pm 0.3 (group B), $p<0.015$. At the end of the study, group A increased sexual activity from 2 sexual encounters per month to 9 , while group B increased from 2 to $6, p<0.021$. 
Conclusions: The combination tadalafil plus ginkgo biloba has a satisfactory therapeutic efficacy on the improvement in the international index of erectile function score, favoring the monthly coital frequency, with time of onset of action and less adverse effects.

Keywords: efficacy; erectile dysfunction; chronic disease; men; therapeutics.

\section{Introducción}

La disfunción eréctil (DE) se define como la incapacidad persistente para alcanzar y mantener una erección adecuada a fin de llevar a cabo una relación sexual satisfactoria ${ }^{(1)}$. Se estima que la disfunción eréctil afecta al 52\% de los hombres entre 40 a 70 años (clasificados de la siguiente manera: 17,2\% leve, $25,2 \%$ moderada y $9,6 \%$ severa), porcentaje que aumenta proporcionalmente con el incremento de la edad y que pasa del $39 \%$ a los 40 años al $67 \%$ a los 70 años ${ }^{(2,3)}$.

La DE se ha reconocido como la primera manifestación clínica y marcador de disfunción endotelial e incluso de enfermedad cardiovascular $^{(4)}$. Las causas más frecuentes involucradas en su etiología son las psicógenas (psicopatías, mala relación de pareja, problemas laborales o sociales, desconocimiento sexual, falsas creencias, malas experiencias sexuales previas, entre otras), enfermedades cardiovasculares y sus factores de riesgo (tabaquismo, hipercolesterolemia, obesidad, entre otros), diabetes, enfermedades prostáticas, depresión, así como muchos de los tratamientos médicos y quirúrgicos que se aplican para resolverlas $^{(5-8)}$. En relación con las enfermedades crónicas más frecuentes, el 15\% de los hombres en tratamiento de hipertensión arterial tiene disfunción eréctil, mientras que la prevalencia en hombres diabéticos oscila entre $40 \%$ y $50 \%$ y en los hombres con dislipidemia la cifra es del $26 \% \%^{(2,9,10)}$.

En la evaluación de la DE se utilizan múltiples cuestionarios, siendo más usada la versión reducida del índice internacional de la función eréctil (International Index of Erectile Funtion [IIEF-5]); un cuestionario validado que consta de cinco preguntas que evalúan cinco aspectos de la función sexual en hombres en los últimos 6 meses, con cinco respuestas posibles por pregunta y con puntajes del 0 al 5 . Se considera la presencia de la DE cuando la puntuación es $\leq 21$ (este punto de corte le permite al cuestionario un $98 \%$ de sensibilidad y $88 \%$ de especificidad para diagnóstico de DE). Se clasifica en cinco categorías: severa (puntuación 5-7), moderada (8-11), media a moderada (12-16), media (17-21), ausencia de DE (22-25) $)^{(1,11)}$.

El objetivo principal en el tratamiento de la disfunción eréctil consiste en detectar la etiología y manejarla, siempre que sea posible, sin enfocarse únicamente en el síntoma. Los factores modificables deben ser abordados antes de utilizar tratamientos farmacológicos o invasivos. El propósito de la estrategia terapéutica dependerá del costo, invasividad y seguridad de la misma, así como de las preferencias del paciente ${ }^{(12)}$.
En el arsenal terapéutico farmacológico de la DE, los inhibidores de la 5-fosfodiesteresa (IPDE-5) (sildenafilo, tadalafilo y vardenafilo) son la primera elección, los cuales refuerzan el efecto del óxido nítrico (ON) e inhiben la fosfodiesterasa del tipo 5 (PDE-5), responsable de incrementar el GMPc en el músculo liso del cuerpo cavernoso; potencian las propiedades vasodilatadoras del ON y producen una vasodilatación prolongada, ya que impiden la hidrólisis en el GMPc, lo que mantiene su efecto en el músculo liso ${ }^{(13-16)}$.

El extracto de ginkgo biloba es un fitofármaco cuyos principales componentes son los flavonoides (ginkgetina, kaempferol, bilobetina, entre otros) y los terpenoides (bilobálidos y ginkgólidos A, B, C y J). Los ginkgólidos son antagonistas del receptor del factor activador de plaquetas (PAF), que ejercen un efecto inhibidor de la agregación plaquetaria. Los flavonoides parecen poseer una función neutralizadora de los radicales libres que favorecen la protección contra los cambios hipóxicos, así como propiedades neuroprotectoras ${ }^{(17-19)}$. Se le han demostrado efectos positivos en el desempeño sexual de personas con patología vasoconstrictiva, dado que influye en los sistemas de óxido nítrico, esto facilita el flujo sanguíneo al generar relajación en el tejido muscular liso, reconociéndosele la factibilidad de que tenga un efecto terapéutico en la disfunción eréctil(20,21).

Los trastornos en la erección son una queja frecuente en hombres con enfermedades crónicas; sin embargo, los IPDE5 se han constituido en la primera opción terapéutica ${ }^{(5,8,13-15)}$. Con base en lo anterior se decidió realizar un estudio en nuestra unidad de sexología para comparar la eficacia terapéutica de dos fármacos (tadalafilo y vardenafilo) en combinación con ginkgo biloba, debido a sus bondades vasodilatadoras dado el influjo en los sistemas de óxido nítrico para el tratamiento de la disfunción eréctil no psicógena en hombres con enfermedades crónicas concomitantes (disfunción eréctil vasculogénica) para observar su respuesta a los medicamentos, así como la posible aparición de efectos adversos.

\section{Material y métodos}

Se realizó un ensayo clínico controlado, enmascarado y aleatorizado, en 56 pacientes de la Clínica Sexológica, en la consulta del autor, entre el 01 de julio de 2013 y el 31 de diciembre de 2016. Participaron hombres diagnosticados con disfunción eréctil moderada o severa de acuerdo con el puntaje del IIEF-5.

\section{Tamaño de la muestra}

No se llevó a cabo un cálculo del tamaño de muestra, sino un muestreo no probabilístico por conveniencia, a partir del universo de pacientes atendidos en la institución participante durante el tiempo del estudio. La selección se hizo mediante una muestra aleatoria simple por medio de una tabla de números aleatorios. 
En el estudio se incluyeron hombres mayores de 45 años con disfunción eréctil no psicógena (vasculogénica) y diagnóstico de hipertensión arterial sistémica de acuerdo con los criterios de JNC 8, con diabetes mellitus tipo 2, dislipidemia e hipotiroidismo como factores de riesgo de disfunción eréctil vasculogénica ${ }^{(22)}$. Se excluyeron a los hombres que presentaron enfermedad coronaria previa, los no controlados con bloqueantes del receptor de la angiotensina (BRA), antecedente de cirugía prostática, trastornos degenerativos hereditarios de retina o retinitis pigmentaria, contraindicación para la medicación del estudio y los que no se interesaron en participar en la investigación.

Los hombres se incluyeron después de aceptar, entender y firmar el consentimiento informado, así como su pareja. Una vez reclutado cada hombre, una enfermera profesional abría de forma consecutiva un sobre opaco con el grupo de estudio y luego le entregaba al paciente el sobre con las tabletas. Los fármacos se prepararon de igual forma, ambos sin características que permitieran evidenciar el medicamento usado. Los hombres fueron tratados con ARA II para la hipertensión, levotiroxina para el hipotiroidismo, metformina para la diabetes y rosuvastatina para la dislipidemia, administradas en dosis única diaria. Los otros medicamentos administrados para el tratamiento de las enfermedades concomitantes fueron ácido acetilsalicílico, furosemida, omeprazol o acetaminofén.

\section{Variables medidas}

Sociodemográficas (edad, raza, escolaridad, estrato socioeconómico, estado civil, ocupación, afiliación al sistema general de seguridad social en salud, convivencia, condición espiritual o religiosa, área de residencia); variables de comportamiento sexual (orientación sexual, edad de la primera relación sexual, número de parejas sexuales, tiempo de convivencia en pareja, pareja con disfunción sexual e infidelidad); sedentarismo, tabaquismo e ingesta de alcohol. Se indagaron, además, las preguntas de los dominios de la encuesta IIFE-5. Se incluyeron dentro de las variables que se tomaron de cada paciente los datos de peso, talla, índice de masa corporal (IMC), presión sistólica, diastólica y frecuencia cardíaca. Los resultados de laboratorios fueron glucemia, $\mathrm{HbA1C}$, colesterol total, triglicéridos, antígeno prostático específico (PSA), TSH, proteína C reactiva ultrasensible (PCR-us) y testosterona total (previos, durante los controles y al final del estudio) y esquemas farmacológicos instaurados.

Las variables de interés primario fueron puntaje del IIFE-5, comportamiento sexual (frecuencia coital mensual), tiempo de respuesta del IPDE-5 (tiempo de inicio de acción), porcentaje de satisfacción de la mejoría de la disfunción y efectos adversos.

\section{Intervención}

A los participantes se les suministró la posología durante los 36 meses del estudio. Se aleatorizaron dos grupos: A (tadalafilo 20 mg más ginkgo biloba $80 \mathrm{mg}$ ) y B (vardenafilo 20 mg más ginkgo biloba $80 \mathrm{mg}$ ). A cada hombre se le aprovisionó la dosis del IPDE-5. El tadalafilo se suministró desde 30 minutos hasta 12 horas antes de la actividad sexual. Se recomendó tragar el comprimido con un vaso de agua, con una pauta de dosificación de cada 2 días. El vardenafilo se suministró a demanda (30 a 60 minutos antes de la actividad sexual). Se recomendó tragar el comprimido con un vaso de agua, con una pauta máxima de dosificación de 1 vez al día y una dosis única diaria de ginkgo biloba en ambos grupos. Se les sugirió a los participantes tomar el tadalafilo o vardenafilo con o sin alimentos, pero preferiblemente no hacerlo después de una comida copiosa o con un alto contenido en grasas. Los controles se realizaron a los $3,6,9,12$, 18, 24, 30 y 36 meses, para un total de 9 controles.

Se evaluó la eficacia terapéutica con el cuestionario de disfunción eréctil: índice internacional de la función eréctil (IIEF-5). La frecuencia coital se evaluó con la pregunta: ¿cuántas veces tuvo relaciones sexuales el mes pasado?" (período de tiempo definido al entrevistado como el lapso de tiempo entre los 30 días anteriores). El tiempo de respuesta se midió entre el momento del estímulo sexual (contacto físico) y la aparición de la erección. La evaluación del porcentaje de satisfacción de la mejoría de la disfunción se evaluó mediante el diseño de una tabla que hacía una evaluación subjetiva de la siguiente manera: satisfactoria (mejoría $>75 \%$ ), buena (mejoría 50-74\%), regular (mejoría 25-49\%) y mala (mejoría <25\%).

\section{Análisis estadístico}

Todos los datos se almacenaron en una tabla de Excel y se tabularon con el mismo programa. Los datos fueron analizados con el programa estadístico SPSS versión 12.0 para Windows. Las variables cuantitativas se presentan como variables continuas mediante medidas de tendencia central y dispersión. Las variables categóricas (frecuencias y proporciones) y continuas (media, desviación estándar [DS]). La comparabilidad inicial entre los dos grupos de estudio se realizó con pruebas paramétricas (distribución t Student) para variables continuas y pruebas no paramétricas $(\chi 2)$ para variables categóricas. Nivel de significación $p \leq 0,05$. Los medicamentos fueron proporcionados por el autor principal, asegurándose de un adecuado suministro a cada paciente.

\section{Aspectos éticos}

La investigación cumplió los requisitos para la investigación en humanos según la Declaración de Helsinki y la Resolución 8430 de 1993 del Ministerio de Salud de Colombia.

\section{Resultados}

Se seleccionaron 56 hombres, de los cuales 28 fueron asignados al grupo A y 28 al grupo B. Durante el seguimiento, 6 participantes del grupo A y 5 del grupo B necesitaron otro tipo de tratamiento antihipertensivo o ajustar la terapia hipogluce- 
miante, por lo que se retiraron del estudio; al final se aplicaron 45 cuestionarios (22 en el grupo A y 23 en el grupo B).

Los participantes de ambos grupos no mostraron diferencias significativas entre sí, ni en cuanto a los factores de riesgo. Las características sociodemográficas de la población de hombres estudiados reportaron que la edad promedio fue de 57,6 (DS $\pm 8,4$ ) años y 58,2 (DS $\pm 7,8$ ) años para cada grupo, respectivamente, rango entre 18 y 72 años ( $p=0,21)$. La mayoría eran hispánicos (55,55\%). El 80\% estaba casado o en unión libre. El
84,44\% profesaba la religión católica. Se encontró educación secundaria en el $57,77 \%$ y el $20 \%$ contaba con título universitario. Respecto al estrato socioeconómico de la población se observó que el $86,66 \%$ era de estrato alto. El 64,44\% era trabajador independiente, el 95,55\% pertenecía al régimen contributivo de seguridad social en salud y el 73,33\% residía en el área urbana. En la tabla 1 se detallan las características sociodemográficas, las condiciones del inicio y las otras variables de control, las cuales fueron similares en ambos grupos.

Tabla 1. Características sociodemográficas y otras variables de control

\begin{tabular}{|c|c|c|c|}
\hline & $\begin{array}{c}\text { Grupo A } \\
n=22\end{array}$ & $\begin{array}{c}\text { Grupo B } \\
n=23\end{array}$ & Valor de $p$ \\
\hline Edad (X $\pm \mathrm{DS})$ & $57,6 \pm 8,4$ & $58 \pm 7,8$ & 0,378 \\
\hline Peso (X $\pm D S)$ & $81,6 \pm 4,8$ & $78,2 \pm 1,6$ & 0,291 \\
\hline Talla $(X \pm D S)$ & $1,74 \pm 0,36$ & $1,75 \pm 0,48$ & 0,156 \\
\hline $\mathrm{IMC}(\mathrm{X} \pm \mathrm{DS})$ & $30,6 \pm 7,2$ & $28,5 \pm 0,9$ & 0,357 \\
\hline \multicolumn{4}{|l|}{ Raza } \\
\hline Afrocolombianos (\%) & 27,27 & 30,43 & 0,579 \\
\hline Hispánicos (\%) & 54,54 & 56,52 & 0,726 \\
\hline Indígenas (\%) & 18,18 & 13,04 & 0,102 \\
\hline \multicolumn{4}{|l|}{ Escolaridad } \\
\hline Estudios primarios (\%) & 27,27 & 21,73 & 0,423 \\
\hline Secundaria (\%) & 54,54 & 60,86 & 0,297 \\
\hline Universitarios (\%) & 18,18 & 21,73 & 0,231 \\
\hline \multicolumn{4}{|l|}{ Estado civil } \\
\hline Casados (\%) & 40,9 & 43,47 & 0,381 \\
\hline Unión libre (\%) & 36,36 & 39,13 & 0,216 \\
\hline Solteros (\%) & 22,72 & 17,39 & 0,168 \\
\hline \multicolumn{4}{|l|}{ Ocupación } \\
\hline Independiente (\%) & 63,63 & 65,21 & 0,852 \\
\hline Empleado (\%) & 36,36 & 34,78 & 0,768 \\
\hline Glucemia (mmol/L) & $4,59 \pm 0,06$ & $4,35 \pm 0,09$ & 0,462 \\
\hline $\mathrm{HbA1C}(\%)$ & $7,29 \pm 2,46$ & $7,74 \pm 6,06$ & 0,279 \\
\hline Colesterol en plasma (mmol/L) & $5,73 \pm 0,18$ & $5,49 \pm 0,15$ & 0,243 \\
\hline Triglicéridos en plasma (mmol/L) & $2,67 \pm 0,06$ & $2,64 \pm 0,15$ & 0,258 \\
\hline PSA (ng/mL) & $3,81 \pm 0,54$ & $3,75 \pm 0,72$ & 0,372 \\
\hline $\mathrm{TSH}(\mathrm{mIU} / \mathrm{L})$ & $5,49 \pm 1,32$ & $5,61 \pm 1,05$ & 0,612 \\
\hline PCR-us (mg/L) & $4,32 \pm 0,78$ & $4,59 \pm 0,84$ & 0,459 \\
\hline Testosterona total (ng/dL) & $579 \pm 1,74$ & $549 \pm 1,38$ & 0,237 \\
\hline Ingesta alcohol (\%) & 45,45 & 43,47 & 0,825 \\
\hline Tabaquismo (\%) & 27,27 & 39,13 & 0,063 \\
\hline Sedentarismo (\%) & 81,81 & 78,26 & 0,684 \\
\hline
\end{tabular}


El 33,33\% era fumador habitual, mientras que $62,22 \%$ se declaró como exfumador. El 44,44\% consumía alcohol. La prevalencia de sedentarismo en la población total fue del 91,11\%. Una alta proporción de los hombres utilizaba un IPDE-5 automedicado (68,88\%), con predominio del sildenafilo de $50 \mathrm{mg}$ a demanda (58,06\%). El 62,22\% manifestó no utilizar el preservativo por temor a perder o no lograr la erección. El 24,44\% afirmó nunca haber utilizado el preservativo. El 71,11\% se mostró insatisfecho con el tamaño y grosor del pene.

El número de parejas sexuales reportó una mediana de 15, con un rango entre 1 y 27 . El 51,11\% refirió más de 10 años de tiempo de convivencia en pareja; e 62,22\% manifestó que la pareja presentaba alguna disfunción sexual; el 13,33\% refirió que la pareja le había sido infiel; el 86,66\% afirmó haber sido infiel por lo menos en una ocasión, mientras el 73,33\% expresó serlo con frecuencia. La orientación sexual es predominantemente heterosexual $(93,33 \%)$. En relación con el inicio de la actividad sexual, la edad promedio es de 15,6 años (DS $\pm 2,4$ ). La práctica sexual más frecuente es el coito vaginal (100\%) y la menos frecuente el coito anal (15,55\%). La masturbación es considerada una práctica poco común en el 75,55\% de los hombres tratados.

A la pregunta ¿cuántas veces tuvo relaciones sexuales el mes pasado? (período de tiempo definido al entrevistado como el lapso de tiempo entre los 30 días anteriores), el 53,33\% del total de la población estudiada informó tener relaciones coitales 4 veces por mes; de estos, el 70,83\% lo hace una vez por encuentro y el 29,16\% lo hace dos veces por encuentro, aunque con dificultad para la erección. El 11,11\% de los hombres informó haber sufrido alguna forma de violencia por parte de la pareja debido a su problema de erección.

La puntuación del IIEF-5 en la población global del total de los 45 hombres fue de 11,49 puntos, siendo el puntaje mayor de 14,28 puntos y el mínimo de 6,24 puntos, con una desviación estándar de $\pm 3,57$ puntos. En el grupo A, el puntaje del IIEF-5 inicial fue de 9,63 $\pm 1,26$ puntos y en el grupo B de 10,35 $\pm 1,59$ puntos $(p=0,324)$. En el grupo $A$, la frecuencia coital mostró una mediana de 2 encuentros sexuales por mes (rango entre 1 y 5) y en el grupo B de 2 encuentros sexuales por mes (rango entre 1 y 4) ( $p=0,0258)$.

$\mathrm{Al}$ ingreso, a la pregunta acerca de la frecuencia para alcanzar la erección el 8,88\% afirmó que la lograba "siempre" con ayuda farmacológica, el 15,55\% dijo que "frecuentemente" (3/5) lograban la erección con medicación y el 68,88\% refirió "ocasionalmente" (1-2/5) a pesar de las circunstancias favorables y uso de medicación. El 4,44\% de los hombres refirió haber asistido a una consulta de sexología; todos afirmaron haber recibido tratamiento farmacológico, sin otro tipo de intervención.

A los 3 meses de seguimiento, los hombres de ambos grupos presentaron mejoras en la puntuación del IIEF-5. En el grupo A, el puntaje del IIEF-5 fue de 17,04 \pm 1,05 puntos, mientras en el grupo B fue de 13,26 $\pm 1,02$ puntos ( $p=0,01)$. La frecuencia coital mostró una mediana de 4 encuentros sexuales por mes (rango entre 2 y 8 ) en el grupo A y de 3 encuentros sexuales por mes (rango entre 1 y 6 ) en el grupo $B$, siendo estadísticamente significativo ( $p>0,045$ ) (tablas 2 y 3). En el tiempo de respuesta, el grupo de tadalafilo reportó menores tiempos $(37,14 \pm 8,25$ minutos mientras vardenafilo arrojó 49,62 \pm 10,29 minutos; $p=0,018$ ); así como en la aparición de efectos adversos, los cuales fueron menores en el grupo del tadalafilo $(25,38 \pm 3,42 \%$ vs. 37,23 $\pm 4,17 \% ; p=0,027)$. El porcentaje de satisfacción reportó un porcentaje del 77,27\% en el grupo A y 60,86\% en el grupo B ( $p=0,012)$.

A los 9 meses se observó un mayor incremento en la puntuación del IIEF-5 en ambos grupos (A: 19,74 \pm 0,6 y B: 16,05 $\pm 0,9)$; $(p=0,0021)$. La frecuencia coital arrojó una mediana de 6 encuentros sexuales por mes (rango entre 3 y 9) en el grupo A y de 4 encuentros sexuales por mes (rango entre 2 y 7 ) en el grupo B, siendo estadísticamente significativo $(p>0,042)$ (tablas 2 y 3). En el tiempo de respuesta, el grupo de tadalafilo reportó menores tiempos $(32,79 \pm 7,32$ minutos mientras vardenafilo mostró 41,79 \pm 9,75 minutos; $p=0,015$ ); así como en la aparición de efectos adversos, siendo menores en el grupo del tadalafilo $(21,9 \pm 3,3 \%$ vs. $30,6 \pm 3,9 \% ; p=0,027)$. El porcentaje de satisfacción reportó 81,81\% en el grupo A y 65,21\% en el grupo B $(p=0,015)$.

A los 18 meses se observó un progresivo incremento en la puntuación del IIEF-5 en ambos grupos (A: 21,54 $\pm 0,3$ y B: $17,94 \pm 0,6$ ); $p=0,0051$. La frecuencia coital reportó una mediana de 7 encuentros sexuales por mes (rango entre 4 y 10) en el grupo A y de 5 encuentros sexuales por mes (rango entre 3 y 8) en el grupo B, con cambios estadísticamente significativos $(p<0,001)$ (tablas 2 y 3). En el tiempo de respuesta, el grupo de tadalafilo reportó menores tiempos $(28,56 \pm 6,12$ minutos mientras vardenafilo mostró 37,41 $\pm 8,31$ minutos; $p=0,006$ ); así como en la aparición de efectos adversos, siendo menores en el grupo del tadalafilo $(19,5 \pm 3,6 \%$ vs. $29,4 \pm 3,3 \% ; p=0,021)$. El porcentaje de satisfacción reportó un porcentaje del 86,36\% en el grupo A y 73,91\% en el grupo B ( $p=0,018)$.

A los 36 meses se observó un persistente aumento en la puntuación del IIEF-5 en ambos grupos (A: 21,78 $\pm 0,9$ y B: $18,57 \pm 0,3) ; p=0,0012$. La frecuencia coital reportó una mediana de 9 encuentros sexuales por mes (rango entre 6 y 12) en el grupo A y de 6 encuentros sexuales por mes (rango entre 4 y 9) en el grupo $\mathrm{B}$, siendo estadísticamente significativos ( $p$ $<0,012$ ) (tablas 2 y 3). En el tiempo de respuesta, el grupo de tadalafilo reportó menores tiempos $(26,13 \pm 5,49$ minutos, mientras vardenafilo mostró 34,86 $\pm 7,26$ minutos; $p=0,003$ ); así como en la aparición de efectos adversos, siendo menores en el grupo del tadalafilo $(18,9 \pm 2,7 \%$ vs. $27,6 \pm 3,6 \%$; $p=$ $0,018)$. El porcentaje de satisfacción reportó ser de 95,45\% en el grupo A y $82,6 \%$ en el grupo B ( $p=0,021)$. 
El promedio del puntaje en el IIEF- 5 antes del inicio del tratamiento fue de $9,63 \pm 1,26$ puntos y a los 36 meses de $21,78 \pm$ 0,9 en el grupo que recibió vardenafilo; de 10,35 $\pm 1,59$ al inicio y de 18,57 $\pm 0,3$ a los 36 meses en el grupo que recibió tadalafilo (tablas 2 y 3 ), en esta observación, la diferencia antes y después de la terapia fue estadísticamente significativa en relación con el inicio del estudio y entre grupos ( $p=0,0001)$.

La frecuencia coital mensual antes del inicio del tratamiento reportó una mediana de 2 encuentros sexuales por mes en ambos grupos. A los 9 meses, el grupo A registró una mediana de 6, mientras el grupo B registró 4 coitos por mes. A los 18 meses, el grupo A registró 7 coitos por mes y el grupo B 5 . A los 36 meses, el grupo A reportó una mediana de 9 coitos mensuales, mientras el grupo B reportó 6 (tablas 2 y 3 ). Se observa que antes y después de la terapia la diferencia fue estadísticamente significativa en relación con el inicio de la investigación y entre grupos $(p<0,01)$.

Se encontraron diferencias significativas, entre los dos grupos en el tiempo de respuesta, que fue mayor en el grupo de vardenafilo desde el inicio $(49,62 \pm 10,29$ minutos $v s$. $37,14 \pm 8,25$ minutos; $p=0,018$ ), manteniéndose así a lo largo del estudio. Igual sucedió con la aparición de efectos adversos, los cuales fueron menores en el grupo del tadalafilo desde el inicio $(25,38 \pm 3,42 \%$ vs. $37,23 \pm 4,17 \%)$ hasta el final $(18,9 \pm$ $2,7 \%$ vs. 27,6 $\pm 3,6 \%$ ); $p=0,027$ (tablas 2 y 3).

En el porcentaje de satisfacción de la mejoría de la disfunción desde el inicio del estudio hasta el final se encontró una diferencia estadísticamente significativa entre los grupos (grupo A [inicio]: 77,27\% vs. grupo B [inicio]: 60,86\%; $p=$ $0,003)$. Al final: grupo A $(95,45 \%)$ y grupo B $(82,6 \%) p<0,027$.

La puntuación final del IIEF-5 en el total de la población de hombres fue de 20,07 \pm 0,9 puntos (rango entre 15,37 y 21,87 puntos). El número de hombres que mejoraron en el IIEF-5 al final del estudio en el grupo A, logrando una puntuación igual o mayor de 17 puntos, fue de 18 pacientes $(81,81 \%)$. En el grupo B fue de 15 pacientes $(65,21 \%)$, diferencia estadísticamente significativa $(p<0,021)$.

Se confirmó que la administración de tadalafilo más ginkgo biloba (grupo A) elevó significativamente el puntaje en el IIEF5 a partir del tercer mes después del inicio de la combinación, en comparación con los del grupo B $(p<0,001)$ (tablas 2 y 3 ). Se presentó un aumento mayor del puntaje en el IIEF-5 en el grupo A de 7,41 puntos a diferencia del grupo B, con un ascenso de 2,91 puntos y una diferencia estadísticamente significativa $(p<0,003)$. Los hombres con diabetes que ingerían alcohol o que fumaban mostraron una mayor disminución en el puntaje del IIFE-5 en comparación con los que no ingerían alcohol o que no fumaban; sin embargo, a pesar de la respectiva diferencia, esta no fue estadísticamente significativa $(p=0,183)$.

Los efectos adversos que se presentaron con mayor frecuencia en el grupo B fueron cefalea, congestión nasal, dispepsia, dolores de espalda, enrojecimiento y mialgias. La mayor diferencia radicó en la cefalea, la cual se presentó en el $26,08 \%$ del grupo B y el $4,54 \%$ en el grupo A $(p<0,006)$; sin encontrarse diferencias estadísticamente significativas en los otros efectos adversos.

Tabla 2. Comportamiento de las variables antes y después del tratamiento con tadalafilo

\begin{tabular}{l|c|c|c|c|c}
\multicolumn{1}{c|}{ Variables } & Inicio & $\mathbf{3}$ meses & $\mathbf{9}$ meses & $\mathbf{1 8}$ meses & $\mathbf{3 6}$ meses \\
\hline IIEF-5 & $9,63 \pm 1,26$ & $17,04 \pm 1,05$ & $19,74 \pm 0,6$ & $21,54 \pm 0,3$ & $21,78 \pm 0,9$ \\
\hline Frecuencia coital mensual & 2 & 4 & 6 & 7 & 9 \\
\hline Tiempo de respuesta (minutos) & & $37,14 \pm 8,25$ & $32,79 \pm 7,32$ & $28,56 \pm 6,12$ & $26,13 \pm 5,49$ \\
\hline Satisfacción (\%) & & 77,27 & 81,81 & 86,36 & 95,45 \\
\hline Efectos adversos & & $25,38 \pm 3,42$ & $21,9 \pm 3,3$ & $19,5 \pm 3,6$ & $18,9 \pm 2,7$ \\
\hline
\end{tabular}

Tabla 3. Comportamiento de las variables antes y después del tratamiento con vardenafilo

\begin{tabular}{l|c|c|c|c|c}
\hline \multicolumn{1}{c|}{ Variables } & Inicio & $\mathbf{3}$ meses & $\mathbf{9}$ meses & $\mathbf{1 8}$ meses & $\mathbf{3 6}$ meses \\
\hline IIEF-5 & $10,35 \pm 1,59$ & $13,26 \pm 1,02$ & $16,05 \pm 0,9$ & $17,94 \pm 0,6$ & $18,57 \pm 0,3$ \\
\hline Frecuencia coital mensual & 2 & 3 & 4 & 5 & 6 \\
\hline Tiempo de respuesta (minutos) & & $49,62 \pm 10,29$ & $41,79 \pm 9,75$ & $37,41 \pm 8,31$ & $32,86 \pm 7,26$ \\
\hline Satisfacción (\%) & & 60,86 & 65,21 & 73,91 & 82,6 \\
\hline Efectos adversos & & $37,23 \pm 4,17$ & $30,6 \pm 3,9$ & $29,4 \pm 3,3$ & $27,6 \pm 3,6$ \\
\hline
\end{tabular}


Al final del estudio no se encontró diferencia estadísticamente significativa en los niveles de glucemia (grupo A 4,32 $\pm 0,48 \mathrm{mmol} / \mathrm{L} v s$. grupo $\mathrm{B}, 4,26 \pm 0,78 \mathrm{mmol} / \mathrm{L})(p=0,528)$. El colesterol y los triglicéridos no mostraron diferencias significativas entre el inicio y el final del estudio, como tampoco entre los grupos.

La cifra de HbA1C al ingreso tuvo una media de 7,29 \pm $2,46 \%$ en el grupo A y 7,74 $\pm 3,06 \%$ en el grupo B ( $p=0,096)$. Al final del estudio se observó un descenso a $6,42 \pm 1,32 \%$ para el grupo A y 6,57 $\pm 1,17 \%$ para el grupo B, no siendo significativas las diferencias en ambos grupos. Se encontró una relación negativa en ambos grupos entre los niveles de $\mathrm{HbA} 1 \mathrm{C}$ y en el puntaje obtenido en el IIFE-5, estadísticamente significativo ( $p=0,001)$.

La PSA inicial fue $3,81 \mathrm{ng} / \mathrm{mL}$ y $3,75 \mathrm{ng} / \mathrm{mL}$ en los grupos A y B, respectivamente ( $p=0,18)$; al final del estudio hubo un descenso hasta $3,27 \mathrm{ng} / \mathrm{mL}$ para el grupo A y 3,39 ng/mL para el grupo B, las diferencias en ambos grupos no son significativas. La TSH presentó un descenso de 7,2 a 5,4 mIU/L y 7,5 a 5,1 $\mathrm{mIU} / \mathrm{L}$ en los grupos A y B, respectivamente, no siendo significativa la diferencia. La PCR-us disminuyó de 4,32 mg/L a 3,66 $\mathrm{mg} / \mathrm{L}$ en el grupo A y de 4,59 mg/L a 3,52 mg/L en el grupo B, no siendo significativa la diferencia entre los grupos; y se mantuvo inferior a $10 \mathrm{mg} / \mathrm{L}$ en ambos grupos al final del estudio. Los niveles séricos de testosterona total promedio en el grupo $\mathrm{A}$ fueron de $579 \pm 32,37 \mathrm{ng} / \mathrm{dL}$ y $549 \pm 22,56 \mathrm{ng} / \mathrm{dL}$ en el grupo B al inicio ( $p=0,33)$, y al final de $582 \pm 41,79 \mathrm{ng} / \mathrm{dL}$ y $567 \pm 52,68$ $\mathrm{ng} / \mathrm{dL}$, respectivamente, sin diferencia significativa.

En relación con el IMC, el peso corporal siempre fue significativamente menor en el grupo B $(72,9 \pm 4,5)$ respecto al grupo A $(80,4 \pm 2,7)$ a partir de los 12 meses de iniciada la terapia $(p<0,001)$. El déficit fue de 6,9 y $10 \%$ a los 18 y 36 meses, respectivamente, en comparación con el grupo A ( $p=$ $0,006)$. El IMC al final fue diferente entre los grupos, 28,17 \pm 2,7 (grupo A) y 24,03 $\pm 4,2$ (grupo B) ( $p=0,003$ ).

Con respecto al comportamiento de la presión sistólica, al comienzo se encontró una presión arterial sistólica basal promedio de 124,29 mm Hg y una basal al final del estudio de 120,75 mm Hg. Por su parte, la presión diastólica al inicio determinó una basal de 72,81 mm Hg y una basal al final de $70,26 \mathrm{~mm} \mathrm{Hg}$. La frecuencia cardíaca al inicio registró una basal de 78,42 latidos/min y una basal al final de 75,72 latidos/ min, no siendo estadísticamente significativo $(p>0,05)$.

Los porcentajes de ingesta de alcohol, tabaquismo y sedentarismo registrados al ingreso del estudio no se mostraron constantes a lo largo del seguimiento, ya que se encontró una reducción cercana al 6\%, pero no mostró diferencias estadísticamente significativas al final del estudio.

\section{Discusión}

En una sociedad patriarcal, como la nuestra, la disfunción eréctil ejerce un impacto negativo tanto en la actividad sexual como en la autoestima, así como en la calidad de vida del in- dividuo, lo que genera mayor dificultad para relacionarse con la pareja ${ }^{(23)}$. El diagnóstico de la disfunción eréctil es y será clínico, siendo relevante conocer los antecedentes médicos y psicológicos de los hombres y sus parejas ${ }^{(24)}$. La anamnesis suele identificar los trastornos que generalmente se asocian con su aparición y el IIFE-5 es, sin lugar a dudas, una excelente herramienta complementaria ${ }^{(1,11)}$.

Los resultados de esta investigación son consistentes con los datos publicados por Reece y colaboradores acerca de que los inhibidores de la 5-fosfodiesterasa (tadalafilo y vardenafilo) favorecen la calidad de las erecciones, mejoran la satisfacción de las relaciones sexuales coitales y disminuyen la severidad de la disfunción eréctil que no ha respondido a la terapia de primera línea con un solo agente ${ }^{(25)}$.

En este estudio, la erección mejoró en el 82,6\% de los hombres tratados con la combinación que incluía vardenafilo, porcentaje similar al $80 \%$ reportado por Goldstein y colaboradores en una población de hombres de iguales características, en donde el vardenafilo mejoró estadísticamente la función eréctil y, en general, fue bien tolerado por los pacientes ${ }^{(26)}$. Nuestros resultados son concordantes con los presentados por Segraves RT y colaboradores, donde los hombres mejoraron la función sexual con $20 \mathrm{mg}$ de tadalafilo al reportar la erección en el $76 \%$ de las ocasiones en los primeros 30 minutos, ya que en el presente estudio se reportó una erección en el 77,27\% de las ocasiones en los primeros 39 minutos ${ }^{(27)}$. Mejoría significativamente mayor a la reportada por Sáenz de Tejada y colaboradores, el cual resalta un $64 \%$ con dosis de $20 \mathrm{mg}^{(28)}$.

A pesar de los buenos resultados obtenidos al final del estudio, donde la satisfacción logró el 95,45\% en los hombres del grupo A y el 82,6\% en los hombres del grupo B, lo que muestra una manifiesta subida en el puntaje del IIFE-5; lo cierto es que ninguno de los participantes logró alcanzar el puntaje $\geq 22$ (pasando de una disfunción eréctil severa a una leve a moderada). No obstante, es significativo el incremento de la puntuación final del IIEF-5 en el total de la población de hombres $(20,07 \pm 0,9$ puntos $)$ frente a la puntuación del inicio (11,49 puntos).

En relación con la frecuencia de las relaciones sexuales, en el grupo A se evidenció un incremento de la mediana al inicio de 2 coitos por mes a 9 al final, mientras que el grupo B pasó de 2 a $6(p<0,01)$. En general, los hombres del grupo A manifestaron mayor satisfacción que los del grupo B con el resultado de la combinación, alentados, además, por los menores efectos adversos, diferencias que al obtener un $p=0,027$ son estadísticamente significativas, tal como lo describieron Giuliano y colaboradores ${ }^{(29)}$.

Una ventaja a resaltar del tadalafilo es que su eficacia puede extenderse 24 a 36 horas después de la ingesta, lo cual favorece la satisfacción debido a su farmacocinética (larga vida media de 17,5 a 22,5 horas), muy superior a la del vardenafilo ( 4 a 5 horas), con una pauta máxima de dosificación de 1 vez al día ${ }^{(30-34)}$. Esto fa- 
vorece la espontaneidad de la actividad coital en horas postreras a su administración y facilita asumir la relación sexual con natural espontaneidad y libre de presiones psicológicas que influyan negativamente en el desempeño sexual del individuo, esto también facilita a la pareja una ventana abierta para la relación con éxito en cualquier momento, lo cual se constituye en un beneficio adicional $^{(35)}$. A diferencia del vardenafilo, el tadalafilo no ve alterada su absorción con los alimentos ni con el alcohol, por tanto, no se afecta su inicio de acción farmacológica, por lo que se logra sostener la efectividad terapéutica demostrada en este estudio ${ }^{(14)}$.

Al tadalafilo se le atribuye un discreto efecto antihipertensivo en los hombres hipertensos, pero nosotros no vimos esa influencia en el grupo A, a pesar de la combinación con ginkgo biloba ${ }^{(36)}$. En esta investigación se evidencia una significativa mejoría en el puntaje del IIFE-5 en grupo del tadalafilo, con diferencias estadísticamente significativas en cuanto al tiempo de respuesta de aparición de la erección y respecto a la tolerancia del tratamiento por períodos largos, sin encontrarse un incremento significativo de los costos.

Los efectos adversos se presentaron con mayor frecuencia en el grupo B, siendo la cefalea el más comúnmente informada, tal como lo reportan otros estudios, lo que prueba que ambos fármacos son bien tolerados ${ }^{(27,37)}$. En ambos grupos se notó una significativa tendencia a alcanzar los valores normales de los parámetros bioquímicos, lo que se explica en el proceso de intervención, puesto que se les educaba en cada consulta acerca de la relevancia de alcanzar las metas. Se reforzaban los hábitos de vida saludables y la importancia de seguir la actividad física. Estos cambios se correlacionaron con logros representativos en la pérdida de peso, así como en el abandono o merma en el tabaquismo y el alcohol.

El estudio tiene limitaciones por el pequeño tamaño de la muestra y porque se realizó en un solo centro de sexología, lo cual genera dudas en la posibilidad de extrapolar los resultados a la población general. Entre las fortalezas del estudio se destaca que es la primera investigación realizada en el país con esta combinación (dos inhibidores de la fosfodiesterasa 5 combinados con ginkgo biloba) en hombres con enfermedades crónicas, que puede ser utilizada en una población de similares características.

Se deja como valor agregado del estudio la posibilidad de realizar una investigación que evalúe un solo fármaco inhibidor de la fosfodiesterasa 5, frente a la combinación con fitoterapia (ginkgo biloba).

\section{Conclusiones}

La combinación de tadalafilo más ginkgo biloba tiene mayor eficacia terapéutica sobre la mejora en la puntuación del IIEF-5 que la combinación vardenafilo más ginkgo biloba. El efecto de la combinación para mejorar el puntaje del IIEF-5 se presenta de manera gradual en el transcurso de los 3 primeros meses de tratamiento. Los inhibidores de la 5-fosfodiesterasa han mejorado radicalmente la terapia de la disfunción eréctil. Su eficacia ha sido demostrada en todo tipo de hombres. Su eficacia y adecuada tolerabilidad, con escasos y transitorios efectos secundarios, le han dado pauta como primera opción de tratamiento por encima de las otras alternativas.

\section{Conflicto de intereses}

Los autores declaran no tener ningún conflicto de intereses.

\section{Financiamiento}

Los autores no reciben ningún patrocinio para llevar a cabo este estudio.

\section{Referencias}

1. Espitia-De La Hoz FJ. Prevalencia de disfunción eréctil en hombres del Quindío, y factores de riesgo asociados. Rev Urol Colomb. 2019;28(2):169-76.

2. Feldman HA, Goldstein I, Hatzichristou DG, Krane RJ, McKinlay JB. Impotence and its medical and psychosocial correlates: results of the Massachusetts male aging study. J Urol. 1994;151(1):54-61.

3. Ponholzer A, Temml C, Mock K, Marszalek M, Obermayr R, Madersbacher S. Prevalence and risk factors for erectile dysfunction in 2869 men using a validated questionnaire. Eur Urol. 2005;47(1):80-5.

4. Yavuzgil 0, Altay B, Zoghi M, Gürgün C, Kayıkçığlu M, Kültürsay H. Endothelial function in patients with vasculogenic erectile dysfunction. Inter J Cardiol. 2005;103(1):19-26.

5. Derby CA, Mohr BA, Goldstein I, Feldman HA, Johannes CB, McKinlay JB. Modifiable risk factors and erectile dysfunction: can lifestyle changes modify risk? Urology. 2000;1;56(2):302-6.

6. Villamil I, Díaz JA, Sánchez J, García F, Saborido J, Iglesias M. Disfunción eréctil: un problema poco valorado en Medicina Interna. Estudio de pacientes y medicación relacionada. An Med Inter (Madrid). 2006;23:115-8.

7. Esposito K, Giugliano F, Di Palo C, Giugliano G, Marfella R, D’Andrea F, et al. Effect of lifestyle changes on erectile dysfunction in obese men: a randomized controlled trial. JAMA. 2004;291(24):2978-84.

8. Corona G, Mannucci E, Mansani R, Petrone L, Bartolini M, Giommi R, et al. Organic, relational and psychological factors in erectile dysfunction in men with diabetes mellitus. Eur Urol. 2004;46(2):222-8.
9. Manrique H, Cornejo P, Arismendiz L, Pamo A. Características clínicas y prevalencia de disfunción eréctil en pacientes con diabetes mellitus 2 . Rev Soc Perú Med Interna. 2002;15(2).

10. Seftel AD, Sun P, Swindle R. The prevalence of hypertension, hyperlipidemia, diabetes mellitus and depression in men with erectile dysfunction. J Urol. 2004;171(6):2341-5.

11. Rosen RC, Cappelleri JC, Smith MD, Lipsky J, Peña BM. Development and evaluation of an abridged, 5-item version of the International Index of Erectile Function (IIEF-5) as a diagnostic tool for erectile dysfunction. Int J Impot Res. 1999;11(6):319-26.

12. Hatzichristou D, Rosen RC, Broderick G, Clayton A, Cuzin B, Derogatis L, et al. Clinical evaluation and management strategy for sexual dysfunction in men and women. J Sex Med. 2004;1(1):49-57.

13. Gresser U, Gleiter CH. Erectile dysfunction: comparison of efficacy and side effects of the PDE-5 inhibitors sildenafil, vardenafil and tadalafil-review of the literature. Eur J Med Res. 2002;7(10):435-46.

14. Montorsi F, Verheyden B, Meuleman E, Jünemann KP, Moncada I, Valiquette $\mathrm{L}$, et al. Long-term safety and tolerability of tadalafil in the treatment of erectile dysfunction. Eur Urol. 2004;45(3):339-45.

15. Crowe SM, Streetman DS. Vardenafil treatment for erectile dysfunction Ann Pharmacother. 2004;38(1):77-85.

16. Eardley I, Donatucci C, Corbin J, El-Meliegy A, Hatzimouratidis K, McVary K, et al. Pharmacotherapy for erectile dysfunction. J Sex Med. 2010;7(1):524-40. 
17. Kang BJ, Lee SJ, Kim MD, Cho MJ. A placebo-controlled, double-blind trial of Ginkgo biloba for antidepressant-induced sexual dysfunction. Hum Psychopharmacol. 2002;17(6):279-84.

18. Klein J, Chatterjee SS, Löffelholz K. Phospholipid breakdown and choline release under hypoxic conditions: inhibition by bilobalide, a constituent of Ginkgo biloba. Brain Res. 1997;755(2):347-50.

19. Janssens D, Remacle J, Drieu K, Michiels C. Protection of mitochondrial respiration activity by bilobalide. Biochem Pharmacol. 1999;58(1):109-19.

20. Cohen AJ, Bartlik B. Ginkgo biloba for antidepressant-induced sexual dysfunction. J Sex Marital Ther. 1998;24(2):139-43.

21. Wheatley D. Triple-blind, placebo-controlled trial of Ginkgo biloba in sexual dysfunction due to antidepressant drugs. Hum Psychopharmacol. 2004;19(8):545-8.

22. Carey RM, Whelton PK; 2017 ACC/AHA Hypertension Guideline Writing Committee. Prevention, Detection, Evaluation, and Management of High Blood Pressure in Adults: Synopsis of the 2017 American College of Cardiology/American Heart Association Hypertension Guideline. Ann Intern Med. 2018;168(5):351-8. doi: 10.7326/M17-3203

23. Espitia-De La Hoz FJ. Mi primer año en La Crónica. $1^{\text {a }}$ edición. Bogotá: Editorial Bolívar. 2016. p.150-1.

24. Davis-Joseph B, Tiefer L, Melman A. Accuracy of the initial history and physical examination to establish the etiology of erectile dysfunction. Urology. 1995;45(3):498-502.

25. Reece C, Kumar R, Nienow D, Nehra A. Extending the rationale of combination therapy to unresponsive erectile dysfunction. Rev Urol. 2007:9(4):197-206.

26. Goldstein I, Young JM, Fischer J, Bangerter K, Segerson T, Taylor T, et al. Vardenafil, a new phosphodiesterase type 5 inhibitor, in the treatment of erectile dysfunction in men with diabetes: a multicenter double-blin placebo-controlled fixed-dose study. Diabetes Care. 2003;26(3):777-83.
27. Segraves RT, Lee J, Stevenson R, Walker DJ, Wang WC, Dickson RA. Tadalafil for treatment of erectile dysfunction in men on antidepressants. J Clin Psychopharmacol. 2007;27(1),62-6.

28. Sáenz de Tejada I, Anglin G, Knight JR, Emmick JT. Effects of tadalafil on erectile dysfunction in men with diabetes. Diabetes Care. 2002;25(12):2159-64.

29. Giuliano F, Sánchez-Ramos A, Löchner-Ernst D, Del Popolo G, Cruz N, Leriche A, et al. Efficacy and safety of Tadalafil in men with erectile dysfunction following spinal cord injury. Arch Neurol. 2007;64(11):1584-92.

30. Martin-Morales A, Haro JM, Beardsworth A, Bertsch J, Kontodimas S, EDOS Group. Therapeutic effectiveness and patient satisfaction after 6 months of treatment with tadalafil, sildenafil, and vardenafil: results from the erectile dysfunction observational study (EDOS). Eur Urol. 2007;51(2):541-50.

31. Coward RM, Carson CC. Tadalafil in the treatment of erectile dysfunction. Ther Clin Risk Manag. 2008;4(6):1315-30.

32. Broderick GA. Oral pharmacotherapy and the contemporary evaluation and management of erectile dysfunction. Rev Urol. 2003;57:S9-S20.

33. Eardley I, Ellis P, Boolell M, Wulff M. Onset and duration of action of sildenafil for the treatment of erectile dysfunction. Br J Clin Pharmacol. 2002;531:61S-5S

34. Sánchez-Cruz JJ, Cabrera-León A, Martín-Morales A, Fernández A, Burgos $\mathrm{R}$, Rejas J. Male erectile dysfunction and health-related quality of life. Eur Urol. 2003;44(2):245-53.

35. Eardley I, Cartledge J. Tadalafil (Cialis) for men with erectile dysfunction Int J Clin Pract. 2002;56(4):300-4.

36. Oelke M, Weiss JP, Mamoulakis C, Cox D, Ruff D, Viktrup L. Effects of tadalafil on nighttime voiding (nocturia) in men with lower urinary tract symptoms suggestive of benign prostatic hyperplasia: a post hoc analysis of pooled data from four randomized, placebo-controlled clinical studies. World J Urol. 2014;32(5):1127-32.

37. Vickers MA, Wright EA. Erectile dysfunction in the patient with diabetes mellitus. Am J Manag Care. 2004;10(1):S3-11. 